\title{
CLASSES OF AUTOMORPHISMS OF FREE GROUPS OF INFINITE RANK
}

\author{
BY
}

\author{
ROBERT COHEN
}

ABSTRACT. This paper is concerned with finding classes of automorphisms of an infinitely generated free group $F$ which can be generated by "elementary" Nielsen transformations. Two different notions of "elementary" Nielsen transformations are explored. One leads to a classification of the automorphisms generated by these transformations. The other notion leads to the subgroup $B$ of $\operatorname{Aut}(F)$ consisting of the "bounded length" automorphisms of $F$. We prove that the class of "bounded 3-length" automorphisms $B_{3}$ and the class of "elementary simultaneous" Nielsen transformations generate the same subgroup of Aut $(F)$. We show that for the class $T$ of automorphisms of " 2 occurring generators", the groups generated by $T \cap B$ and the "elementary simultaneous" Nielsen transformations are identical. These results lead to the conjecture that $B$ is generated by the "elementary simultaneous Nielsen transformations".

A study is also made of the subgroup $S$ of the "triangular automorphisms" of $F_{\infty}$, the free group on a countably infinite set of free generators. It is found that a "triangular automorphism" may be factored into "splitting automorphisms" of $F_{\infty}$, which may be viewed as the "elementary" automorphisms of $S$.

1. Introduction. The automorphism group, Aut $(F)$, of a free group $F$ of finite rank has been studied by Jacob Nielsen in his fundamental papers [4], [5] where he succeeded in obtaining a presentation for $\operatorname{Aut}(F)$ by using "elementary" (Nielsen) automorphisms for $F$ which have simple defining relations. The part of his results showing that $\operatorname{Aut}(F)$ is generated by these elementary automorphisms depends on a reduction process applied to a finite set of generators of $F$ where a length function is minimized (see e.g. Magnus-Karrass-Solitar [3, \$3.2], or Hall [1]). In his study of primitive elements in a free group, J. H. C. Whitehead (see [8], [9]) introduced a new set of transformations, the so-called "elementary Whitehead T-transformations", which properly contain the Nielsen transformations. When $\operatorname{rank}(F)$ is finite, Whitehead's automorphisms generate $\operatorname{Aut}(F)$, and it generally takes fewer $T$-automorphisms than elementary Nielsen automorphisms to express $\alpha \in \operatorname{Aut}(F)$ as a product of elementary automorphisms (see E. S. Rapaport [6]). Whitehead's results depend on the length of a finite subset of elements

Received by the editors November 19, 1971.

AMS (MOS) subject classifications (1970). Primary 20E99; Secondary $20 \mathrm{~F} 55$.

Key words and phrases. Free group, automorphism, Nielsen reduction, elementary Nielsen transformation, elementary simultaneous Nielsen transformation, triangular automorphism, splitting automorphism, bounded automorphism. 
of $F$. When $F$ is a free group of infinite rank, the methods of Nielsen and Whitehead do not tend to reduce an infinite system of generators of $F$ in a finite number of steps (see Example 4.5 and Theorem 4.4 below). In order to obtain extensions of Grusko's theorem to infinitely generated groups, D. H. Wagner (see [7]) extended the Nielsen transformations so as to apply to infinite subsets of a group. Wagner's $\tau$-transformations become our elementary simultaneous transformations when applied to a free system $X$ of generators of $F$, where the generators in $\tau(X)$ have uniformly bounded lengths. In $\$ 4$, we study two different notions of elementary Nielsen transformations and their associated automorphisms which agree with the usual operations in the finite rank situation.

Although it is false that $\operatorname{Aut}(F)$ is generated by the elementary simultaneous Nielsen automorphisms as defined in $\$ 4$ when $F$ is a free group of infinite rank, it has been conjectured (Donald Solitar) that the se elementary automorphisms generate the subgroup $B$ of the bounded automorphisms of $F$ (see $\$ 4$ ). The conjecture still stands, but it is proved that the class of "bounded 3" automorphisms is contained in the group generated by these automorphisms. A reduction process, independent of the rank of $F$, is applied to a system of free generators of the required type in order to produce this result.

In $\$ 2$, we produce Theorem 2.2 which becomes a useful tool in the study of the subgroup $S$ of triangular automorphisms of $F_{\infty}$, the free group on a countably infinite set of free generators as defined in $\$ 3$. We find that $S$ is generated by what we call the splitting automorphisms of $F_{\infty}$. A splitting automorphism a may be viewed as a triangular automorphism defined on $F=\Pi_{j \in Z_{+}}^{*}\left\langle A_{j}\right\rangle$ where $\left\langle A_{j}\right\rangle$ is a free group on finitely many generators such that $\alpha_{\text {restricted to }}\left\langle A_{j}\right\rangle$ is a triangular automorphism of $\left\langle A_{j}\right\rangle$, for $j \in \mathbf{Z}_{+}=$positive integers.

In $\$ 5$ we consider a special class $T \subset \operatorname{Aut}(F)$ which is of interest in that the class $T \cap B$ may be generated by the elementary simultaneous Nielsen automorphisms.

The author wishes to express his thanks to Professor Donald Solitar who suggested this area of investigation, and to the referee who brought D. H. Wagner's results to the author's attention.

2. Restricted automorphisms. In this section we obtain a general result on the restriction of an automorphism of $F$ to a subgroup $A \subset F$ generated by a subset of free generators of $F$. We use this result in the remainder of this section to obtain specific information on special automorphisms of $F_{\infty}$, the free group on a countably infinite set of free generators.

2.1. Lemma. Let $F=C * D=C^{\prime} * D$ where $C^{\prime} \subseteq C$. Then $C^{\prime}=C$.

Proof. Consider the endomorphism $\phi: F \rightarrow F$ given by the trivial map on $D$, $\phi: D \rightarrow 1$, and the identity map on $C, \phi: C \stackrel{i}{\rightarrow} C$. Then $\phi: H=C * D \rightarrow C$ is 
onto, while $\phi: H=C^{\prime} * D \rightarrow C^{\prime}$ is also onto. Thus $C=$ range $\phi=C^{\prime}$.

2.2. Theorem. Let $F$ be the free group freely generated by the set $X$ and let $F=A * B$ be a free factorization such that $X \subset A \cup B$.

Suppose $\alpha \in \operatorname{Aut}(F)$ is such that $\left.a\right|_{A}$ is an endomorphism of $A$. Then $\left.a\right|_{A} \epsilon$ $\operatorname{Aut}(A)$ if either rank $A$ or rank $B$ is finite.

Proof. If rank $B$ is finite, we let $A_{1} \subset X \cap A$ be the finite set of generators in $X \cap A$ which occur in the freely reduced elements in the set $\alpha(X \cap B)$. Using elementary inclusion arguments we can show

(i) $F$ is generated by the set $(X \cap A) \cup \alpha(X \cap B)$,

(ii) let $G=\left\langle A_{1}, a(X \cap B)\right\rangle$, then $G=\left\langle A_{1}, X \cap B\right\rangle$,

(iii) the set $A_{1} \cup \alpha(X \cap B)$ freely generates the subgroup $G$,

(iv) $F$ is freely generated by $(X \cap A) \cup \alpha(X \cap B)$,

(v) $A=a(A)$.

We show that (v) follows from (iv). By (iv) we have $F=A * B=A * \alpha(B)=$ $\alpha(A) * \alpha(B)$, and using Lemma 2.1 it follows that $A=\alpha(A)$. Thus $\left.\alpha\right|_{A} \in \operatorname{Aut}(A)$ if rank $B$ is finite.

Next, suppose rank $A$ is finite. By a result of D. H. Wagner [7, Theorem 4.3, p. 374] applied to automorphisms of a free group, there exists a set $Y$ freely generating $F$ satisfying the conditions $X \cap \alpha^{-1}(A \cup B) \subset Y$ and $\alpha(Y) \subset A \cup B$ (Wagner's result is true independent of the ranks of $A$ and $B)$. Putting $Y_{1}=\{y \in Y \mid$ $\alpha(y) \in A\}$ and $Y_{2}=Y-Y_{1}$, we see that $X \cap A \subset Y_{1}, a\left(Y_{1}\right) \subset A$ and $\alpha\left(Y_{2}\right) \subset B$. Since the set $\alpha\left(Y_{1}\right) \cup \alpha\left(Y_{2}\right)$ freely generates $F$, it follows that $\alpha\left(Y_{1}\right)$ and $\alpha\left(Y_{2}\right)$ freely generate $A$ and $B$ respectively. Also, since rank $A$ is finite, $\alpha\left(Y_{1}\right)$ (and thus $Y_{1}$ ) has the same number of generators as $X \cap A$. We conclude that $X \cap A=Y_{1}$ and hence $A=\left\langle\alpha\left(Y_{1}\right)\right\rangle=\langle\alpha(X \cap A)\rangle=\alpha(A)$, so that $\left.\alpha\right|_{A}$ is an automorphism of $A$.

The finiteness restriction cannot be deleted as the following example shows. Consider the free group $F_{\infty}=\left\langle a_{1}, a_{2}, \ldots\right\rangle *\left\langle b_{1}, b_{2}, \ldots\right\rangle$ on the free generators $\left\{a_{1}, a_{2}, \cdots, b_{1}, b_{2}, \cdots\right\}$ and define an automorphism $a$ by the map of the generators given by

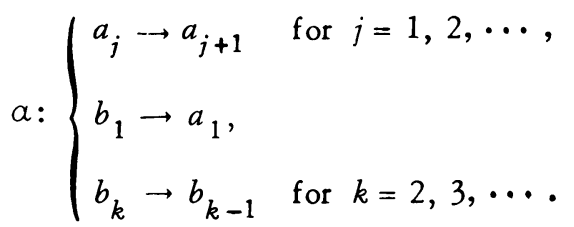

Clearly $\alpha(A) \subset A=\left\langle a_{1}, a_{2}, \ldots\right\rangle$ and $a_{1} \notin \alpha(A)$.

2.3. Corollary. Let $a: F_{\infty} \rightarrow F_{\infty}$ be an automorphism of $F_{\infty}$, the free group on a countably infinite set of free generators $\left\{x_{1}, x_{2}, x_{3}, \cdots\right\}$, such that $\alpha\left(x_{n}\right) \in$ $\left\langle x_{1}, x_{2}, \cdots, x_{n}\right\rangle$ for $n=1,2, \cdots$. Then $\alpha\left(x_{n}\right)=A_{n} x_{n}^{\epsilon_{n}} B_{n}$ where $A_{n}, B_{n} \in\left\langle x_{1}\right.$, $\left.x_{2}, \cdots, x_{n-1}\right\rangle$, for $n=2,3, \cdots$, and $A_{1}=B_{1}=1, \epsilon_{n}= \pm 1$. 
The proof follows by induction on $n$, appealing to Theorem 2.2 and properties of a Nielsen reduced set. Note that the converse of Corollary 2.3 is true and since the proof is trivial, it is omitted.

2.4. Theorem. Let $a \in \operatorname{Aut}\left(F_{\infty}\right)$ be such that $a\left(x_{n}\right) \in\left\langle x_{n}, x_{n+1}, \cdots\right\rangle=G_{n}$, for $n=1,2,3, \cdots$. Then $\left.\alpha\right|_{G_{n}}$ is an automorphism of $G_{n}$; also $\alpha\left(x_{n}\right)=A_{n}{ }_{n}{ }_{n}^{{ }^{n}}{ }_{n} B_{n}$ where $A_{n}, B_{n} \in G_{n+1}$, and $\epsilon_{n}= \pm 1$, for $n=1,2,3, \cdots$.

Proof. To show that $\left.a\right|_{G_{n}}$ is a restricted automorphism, one need only appeal to Theorem 2.2. We show that $\alpha\left(x_{n}\right)=A_{n}{ }_{n}{ }_{n}{ }_{n} B_{n}$.

Note that $\left\langle a\left(x_{n}\right), x_{n+1}, x_{n+2}, \cdots\right\rangle=G_{n}$. This follows from the inclusions

$$
\begin{aligned}
\left.\iota^{\prime} \alpha\left(x_{n}\right), \alpha\left(x_{n+1}\right), \alpha\left(x_{n+2}\right), \cdots\right\rangle & \subseteq\left\langle\alpha\left(x_{n}\right), x_{n+1}, x_{n+2}, \cdots\right\rangle \\
& \subseteq\left\langle x_{n}, x_{n+1}, x_{n+2}, \cdots\right\rangle=G_{n}
\end{aligned}
$$

and the fact that $G_{n}=\left\langle\alpha\left(x_{n}\right), \alpha\left(x_{n+1}\right), \cdots\right\rangle$, since $\left.\alpha\right|_{G_{n}}$ is an automorphism. Now, let $\left\{a\left(x_{n}\right), x_{n+1}, \cdots, x_{m}\right\}$ be a finite subset of these generators such that $x_{n} \in\left\langle\alpha\left(x_{n}\right), x_{n+1}, \cdots, x_{m}\right\rangle(m>n)$. [Clearly, $\alpha\left(x_{n}\right)$ must appear in such a subset of generators.]

By applying elementary Nielsen transformations, we may convert $\left\{\alpha\left(x_{n}\right)\right.$, $\left.x_{n+1}, \cdots, x_{m}\right\}$ to $\left\{W_{n}^{\prime}, x_{n+1}, \cdots, x_{m}\right\}$ so that neither the initial nor terminal element of $W_{n}^{\prime}$ is a symbol in $\left\{x_{n+1}, \cdots, x_{m}\right\} \cup\left\{x_{n+1}^{-1}, \cdots, x_{m}^{-1}\right\}$. Then $\left\{W_{n}^{\prime}, x_{n+1}\right.$, $\left.\cdots, x_{m}\right\}$ is Nielsen reduced, so that $x_{n}$ is a product of these elements. (Clearly, $W_{n}^{\prime}$ must occur in the product.) By the property of a Nielsen reduced system, we have $1=L_{x}\left(x_{n}\right) \geq L_{x}\left(W_{n}^{\prime}\right)$ so that $W_{n}^{\prime}=x_{n}{ }_{n}^{n}$ with $\epsilon_{n}= \pm 1$. It follows that $\alpha\left(x_{n}\right)=$ $A_{n}{ }_{n}{ }_{n}{ }^{n} B_{n}$ where $A_{n}, B_{n} \in\left\langle x_{n+1}, x_{n+2}, \cdots\right\rangle=G_{n+1}$.

3. Triangular and splitting automorphisms. We now confine our attention to the automorphisms of $F_{\infty}$ which were described in Theorem 2.4 , where $F_{\infty}$ is freely generated by $\left\{a_{n} \mid n \in \mathbf{Z}_{+}\right\}$.

3.1. Definition. Let $S=\left\{\alpha \in \operatorname{Aut}\left(F_{\infty}\right) \mid \alpha\left(a_{n}\right) \in\left\langle a_{n}, a_{n+1}, \cdots\right\rangle\right.$ for $\left.n \in \mathbf{Z}_{+}\right\}$. The collection $S$ will be called the group of triangular automorphisms. [ $S$ is indeed a group under composition; that $a \in S$ implies $a^{-1} \in S$ follows by Theorem 2.2.]

We shall characte rize the elements of $S$ in terms of more "elementary" types of triangular automorphisms, the "splitting automorphisms".

3.2. Definition. Let $\gamma$ be an automorphism of $F_{\infty}$ of the following type:

(1) There is a partition $\left\{A_{j} \mid j \in \mathbf{Z}_{+}\right\}$of the generators $\left\{a_{1}, a_{2}, \cdots, a_{n}\right.$, $\cdots\}$ such that $A_{j}=\left\{a_{n_{j}}, a_{n_{j}+1}, \cdots, a_{n_{j+1}-1}\right\}$, where $j \in \mathbf{Z}_{+}$and $n_{1}=1<n_{2}<$ $\cdots<n_{j}<\cdots$.

(2) For $k$ such that $n_{j} \leq k \leq n_{j+1}-1, \gamma\left(a_{k}\right) \in\left\langle a_{k}, a_{k+1}, \cdots, a_{n_{j+1}-1}\right\rangle$. 
An automorphism $\gamma$ satisfying conditions (1) and (2) will be called a "splitting automorphism".

If we write $F_{\infty}=\Pi_{j \in Z_{+}}^{*}\left\langle A_{j}\right\rangle$ (free product) and define the automorphisms $\gamma_{j}$ such that $\gamma_{j}=\left.\gamma\right|_{\left\langle A_{j}\right\rangle}$ on $\left\langle A_{j}\right\rangle$ while $\gamma_{j}$ is defined as the identity on $\left\langle A_{l}\right\rangle$ where $l \neq j$, then we may express $\gamma$ as a product $\gamma=\Pi_{j=1}^{\infty} \gamma_{j}$, where there is no ambiguity in this representation, since all but a finite number of these automorphisms $\gamma_{j}$ act like the identity when applied to a particular freely reduced word in $F_{\infty}$.

We now show the connection between the triangular automorphisms and the splitting automorphisms of $F_{\infty}$.

3.3. Theorem. The group $S$ of triangular automorphisms of $F_{\infty}$ is generated by the splitting automorphisms of $F_{\infty}$. In particular, there exist two splitting automorphisms $\gamma_{1}, \gamma_{2}$ such that $\alpha=\gamma_{1} \cdot \gamma_{2}$ where $\alpha \in S$.

Proof. Let $\alpha \in S$ be a triangular automorphism. We shall write $a\left(a_{j}\right)=W\left(a_{j}\right)$ which is the freely reduced form of $a\left(a_{j}\right)$; notice that the earliest occurring " $a$ " generator in $W\left(a_{j}\right)$ is indeed $a_{j}$ (earliest in the sense of the ordering $a_{1}<a_{2}<$ ...); this follows from Theorem 2.4.

We now proceed in constructing the desired splitting automorphisms..

Step I. Consider the mapping $a_{j} \rightarrow W\left(a_{j}\right)$ for $1 \leq j \leq n$ where $n$ is arbitrary but fixed, and let $b_{1}, \cdots, b_{m}$ be the generators (aside from $a_{1}, \cdots, a_{n}$ ) occurring in the words $W\left(a_{1}\right), \cdots, W\left(a_{n}\right)$. Extend the set $\left\{b_{1}, \cdots, b_{m}\right\}$ to the smallest set of generators $\left\{b_{1}^{\prime}, \cdots, b_{\mu}^{\prime}\right\} \supseteq\left\{b_{1}, \cdots, b_{m}\right\}$ such that the set $\left\{a_{1}, \cdots, a_{n}\right.$, $\left.b_{1}^{\prime}, \cdots, b_{\mu}^{\prime}\right\}$ has no gap in the ordering of the generators [e.g., if say $n=3$, and $\left\{b_{1}, \cdots, b_{m}\right\}=\left\{a_{4}, a_{7}, a_{16}\right\}$, then $\left.\left\{b_{1}^{\prime}, \cdots, b_{\mu}^{\prime}\right\}=\left\{a_{4}, a_{5}, \cdots, a_{14}, a_{16}\right\}\right]$. Here $b_{\mu}^{\prime}$ is the largest occurring generator in the set $\left\{b_{1}, \cdots, b_{m}\right\}$.

Extend the map to

$$
\begin{cases}a_{j} \longrightarrow W\left(a_{j}\right), & 1 \leq j \leq n, \\ b_{k}^{\prime} \longrightarrow b_{k}^{\prime}, & 1 \leq k \leq \mu .\end{cases}
$$

Step II. Let $V_{1}, \cdots, V_{p}$ be the " $W$ " words which generate $b_{1}^{\prime}, \cdots, b_{\mu}^{\prime}$ (aside from $W\left(b_{1}^{\prime}\right), \cdots, W\left(b_{\mu}^{\prime}\right)$ ). Let $c_{1}$ be such that $V_{1}=W\left(c_{1}\right), \cdots, c_{p}$ be such that $V_{p}=W\left(c_{p}\right)$. Extend this set to $\left\{c_{1}^{\prime}, \cdots, c_{\pi}^{\prime}\right\} \supseteq\left\{c_{1}, \cdots, c_{p}\right\}$ so that no gaps occur in the ordered set $\left\{b_{1}^{\prime}, \cdots, b_{\mu}^{\prime}, c_{1}^{\prime}, \cdots, c_{\pi}^{\prime}\right\} ; c_{\pi}^{\prime}$ is the largest occurring generator among the members of $\left\{c_{1}, \cdots, c_{p}\right\}$.

Extend the map to

$$
\begin{cases}a_{j} \longrightarrow W\left(a_{j}\right), & 1 \leq j \leq n, \\ b_{k}^{\prime} \longrightarrow b_{k}^{\prime}, & 1 \leq k \leq \mu, \\ c_{l}^{\prime} \longrightarrow W\left(c_{l}^{\prime}\right), & 1 \leq l \leq \pi .\end{cases}
$$


Next, let $\left\{d_{1}, \cdots, d_{r}\right\}$ be the generators occurring in $W\left(c_{1}^{\prime}\right), \cdots, W\left(c_{\pi}^{\prime}\right)$ not already in $\left\{c_{1}^{\prime}, \cdots, c_{\pi}^{\prime}\right\}$ and proceed, as in Step I, to extend this set to $\left\{d_{1}^{\prime}, \cdots, d_{\rho}^{\prime}\right\} \supseteq$ $\left\{d_{1}, \cdots, d_{r}\right\}$ where no gaps occur in $\left\{c_{1}^{\prime}, \cdots, c_{\pi}^{\prime}, d_{1}^{\prime}, \cdots, d_{\rho}^{\prime}\right\}$. Then extend the map so that $d_{j}^{\prime} \rightarrow d_{j}^{\prime}$, for $j=1,2, \cdots, \rho$. Continue this construction indefinite $: y$.

The above process defines a mapping $N_{A}$ of the free generators of $F_{\infty}$ into $F_{\infty}$ such that

$$
\begin{array}{r} 
\begin{cases}a_{j} \longrightarrow W\left(a_{j}\right), & 1 \leq j \leq n, \\
b_{k}^{\prime} \longrightarrow b_{k}^{\prime}, & 1 \leq k \leq \mu,\end{cases} \\
N_{A}: \begin{cases}c_{l}^{\prime} \longrightarrow W\left(c_{l}^{\prime}\right), & 1 \leq l \leq \pi, \\
d_{q}^{\prime} \longrightarrow d_{q}^{\prime}, & 1 \leq q \leq \rho,\end{cases} \\
\begin{cases}e_{r}^{\prime} \longrightarrow\left(e_{r}^{\prime}\right), & 1 \leq r \leq \sigma, \\
f_{s}^{\prime} \longrightarrow f_{s}^{\prime}, & 1 \leq s \leq \tau,\end{cases}
\end{array}
$$

where the $e_{r}^{\prime}, f_{s}^{\prime}, W\left(e_{r}^{\prime}\right), W\left(f_{s}^{\prime}\right)$ etc., are defined similarly. It is clear that this mapping defines a splitting automorphism of $F_{\infty}$, since the generators in each of the braces in the above are mapped into themselves in the required manner.

We now consider the mapping $N_{B}$ of $F_{\infty}$ given by

$$
\begin{aligned}
& {\left[W\left(a_{j}\right) \longrightarrow W\left(a_{j}\right), \quad 1 \leq j \leq n,\right.} \\
& N_{B}:\left[\begin{array}{ll}
W\left(b_{k}^{\prime}\right) \longrightarrow b_{k}^{\prime}, & 1 \leq k \leq \mu, \\
W\left(c_{l}^{\prime}\right) \longrightarrow W\left(c_{l}^{\prime}\right), & 1 \leq l \leq \pi,
\end{array}\right. \\
& {\left[\begin{array}{ll}
W\left(d_{q}^{\prime}\right) \longrightarrow d_{q}^{\prime}, & 1 \leq q \leq \rho, \\
W\left(e_{r}^{\prime}\right) \longrightarrow W\left(e_{r}^{\prime}\right), & 1 \leq r \leq \sigma,
\end{array}\right.}
\end{aligned}
$$

Using the "W" elements as free generators of $F_{\infty}$, each of the transformations which have been blocked above define an automorphism of the free group generated by the " $W$ " symbols in the block.

For example, to show that the mapping

$$
\begin{array}{ll}
W\left(b_{k}^{\prime}\right) \longrightarrow b_{k}^{\prime}, & 1 \leq k \leq \mu, \\
W\left(c_{l}^{\prime}\right) \longrightarrow W\left(c_{l}^{\prime}\right), & 1 \leq l \leq \pi,
\end{array}
$$


defines an automorphism of the free group $\left\langle W\left(b_{1}^{\prime}\right), \cdots, W\left(b_{\mu}^{\prime}\right), W\left(c_{1}^{\prime}\right), \cdots, W\left(c_{\pi}^{\prime}\right)\right\rangle$, we need only show that $\left\langle W\left(b_{1}^{\prime}\right), \cdots, W\left(b_{\mu}^{\prime}\right), W\left(c_{1}^{\prime}\right), \cdots, W\left(c_{\pi}^{\prime}\right)\right\rangle=\left\langle b_{1}^{\prime}, \cdots, b_{\mu}^{\prime}\right.$, $\left.W\left(c_{1}^{\prime}\right), \cdots, W\left(c_{\pi}^{\prime}\right)\right\rangle$.

Now since $\alpha^{-1} \in S$, by Theorem 2.4 it follows that $b_{k_{i}}^{\prime}=C_{k} W^{\epsilon} \epsilon_{k}\left(b_{k}^{\prime}\right) D_{k}$ where $C_{k}$ and $D_{k}$ are freely reduced words in the generators $W\left(b_{k+1}^{\prime}\right), \cdots, W\left(b_{\mu}^{\prime}\right)$, $W\left(c_{1}^{\prime}\right), \cdots, W\left(c_{\pi}^{\prime}\right), \cdots$ for $1 \leq k<\mu$ and $b_{\mu}^{\prime}=C_{\mu} W^{\epsilon}{ }_{\mu}^{\prime}\left(b_{\mu}^{\prime}\right) D_{\mu}$ where $C_{\mu}$ and $D_{\mu}$ are freely reduced in $W\left(c_{1}^{\prime}\right), W\left(c_{2}^{\prime}\right), \cdots, W\left(c_{\pi}^{\prime}\right), \cdots$. By the choice of $W\left(c_{1}^{\prime}\right), \cdots$, $W\left(c_{\pi}^{\prime}\right)$ it follows that $C_{\mu}, D_{\mu} \in\left\langle W\left(c_{1}^{\prime}\right), \cdots, W\left(c_{\pi}^{\prime}\right)\right\rangle$ and $C_{k}, D_{k} \in\left\langle W\left(b_{k+1}^{\prime}\right), \cdots\right.$, $\left.W\left(b_{\mu}^{\prime}\right), W\left(c_{1}^{\prime}\right), \cdots, W\left(c_{\pi}^{\prime}\right)\right)$. Hence it follows, by applying Nielsen transformations, that

$$
\begin{aligned}
\left\langle b_{1}^{\prime}, \ldots, b_{k}^{\prime}, \cdots, b_{\mu}^{\prime}, W\left(c_{1}^{\prime}\right), \ldots, W\left(c_{\pi}\right)\right\rangle \\
\quad=\left\langle C_{1} W^{\epsilon}{ }^{1}\left(b_{1}^{\prime}\right) D_{1}, \ldots, C_{k} W^{\epsilon}\left(b_{k}^{\prime}\right) D_{k}, \ldots, C_{\mu} W^{\epsilon}\left(b_{\mu}^{\prime}\right) D_{\mu}, W\left(c_{1}^{\prime}\right), \ldots, W\left(c_{\pi}^{\prime}\right)\right\rangle \\
\quad=\left\langle W^{\epsilon}\left(b_{1}^{\prime}\right), \ldots, W^{{ }^{k}}\left(b_{k}^{\prime}\right), \ldots, W^{\epsilon}\left(b_{\mu}^{\prime}\right), W\left(c_{1}^{\prime}\right), \ldots, W\left(c_{\pi}^{\prime}\right)\right\rangle
\end{aligned}
$$

which is essentially what is needed to be shown.

Returning to the automorphisms $N_{A}$ and $N_{B}$ we have $N_{B}\left(W_{\nu}\right)=N_{A}\left(a_{\nu}\right)$. Thus $W_{\nu}=\left(N_{B}^{-1} N_{A}\right)\left(a_{\nu}\right)=N_{A B-1}\left(a_{\nu}\right)=\alpha\left(a_{\nu}\right)$ so that $\alpha=A B^{-1}$ where $A, B^{-1}$ are splitting automorphisms. Setting $\gamma_{1}=A, \gamma_{2}=B^{-1}$, we have $a=\gamma_{1} \cdot \gamma_{2}$ and the theorem is proved.

\section{Elementary simultaneous Nielsen transformations and bounded automor-} phisms. In this section we develop two alternate ways of generalizing the elementary Nielsen transformations so as to operate on collections of $F$ containing an infinite number of elements. One of these notions turns out to be nonlucrative, as Theorem 4.4 below shows. The other notion is sufficiently strong as to lead to the "bounded" automorphism conjecture and to Theorem 4.6, the verification of this conjecture for "bounded 3" automorphisms.

Let $F$ be the free group on the free generators $\left\{x_{\nu} \mid \nu \in \Re\right\}$ where $\Re$ is a linearly ordered indexing set. Let $\left\{W_{\nu} \mid \nu \in \mathcal{N}\right\}=W$ be a collection of freely reduced words in the $x_{\nu}$ generators.

4.1. Definition. The following transformations $\tau_{1}, \tau_{2}$ of the set 60 will be called "elementary simultaneous" Nielsen transformations of rank $|\Re|$ :

1. Permute the $W_{\nu}$ words and take inverses of some of them, i.e., let $\pi$ be a

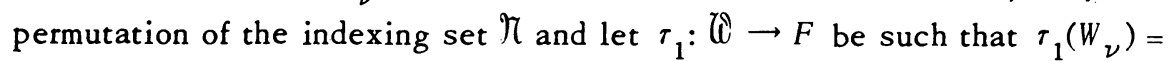
$W_{\pi(\nu)}^{\epsilon \nu}$ where $\epsilon_{\nu}= \pm 1, \nu \in \mathcal{K}$.

2. Let $\tau_{2}: W \rightarrow F$ be a function from 78 to $F, C=\left\{W \in W \mid \tau_{2}(W)=W\right\}$ and $\tau_{2}(U)=U^{ \pm 1} V^{ \pm 1}$ or $V^{ \pm 1} U^{ \pm 1}$ where $V \in \mathcal{C}$ for $U \in \mathbb{C}-\mathcal{C}$. Those words in $\mathcal{C}$ which occur in $\tau_{2}(U)$ for some $U \in \mathcal{C}-\mathcal{C}$ will be denoted by $C^{\prime}$; they are the "active" elements of $\tau_{2}$.

4.2. Definition. Transformations of type $\tau_{1}$ and $\tau_{2}$ where $C^{\prime}$ has finite 
cardinality (or equivalently $\operatorname{card}\left(\mathcal{C}^{\prime}\right)=1$ ) will be called elementary Nielsen transformations .

It is clear that if 10 generates a subgroup $H$ of $F$ then applying an elementary (simultaneous) Nielsen transformation produces another system $\tau(\mathbb{l})$ of generators of the subgroup $H$.

We shall be concerned with the automorphisms induced by the elementary simultaneous transf ormations and the "bounded" automorphisms of $F$ which we now define.

4.3. Definition. Let $a \in \operatorname{Aut}(F)$ be such that there exists some natural number $N$ so that

(i) If $a\left(x_{\nu}\right)=W_{\nu}$ is freely reduced in the $x$ generators, then $L_{x}\left(W_{\nu}\right) \leq N$ for all $\nu \in \Re$ where $L_{x}\left(W_{\nu}\right)$ is the $x$ length of the word $W_{\nu}$.

(ii) If $\alpha^{-1}\left(x_{\nu}\right)=U_{\nu}$ is freely reduced in the $x$ generators, then $L_{x}\left(U_{\nu}\right) \leq N$ for all $\nu \in \pi$. (Equivalently, if $x_{\nu}$ is expressed as a freely reduced expression in the $W_{\nu}$ generators then $L_{w}\left(x_{\nu}\right) \leq N$.)

An automorphism $\alpha$ satisfying conditions (i) and (ii) will be called a bounded $N$ automorphism of $F$. For each fixed natural number $N$, we shall denote by $B_{N}$ the class of all bounded $N$ automorphisms of $F$. The collection $B=\bigcup_{N=1}^{\infty} B_{N}$ clearly forms a subgroup of $\operatorname{Aut}(F)$ which we call the group of bounded automorphisms of $F$.

If $F$ is the free group on the free generators $x_{1}, x_{2}, \cdots, x_{n}$ where $n$ is finite, then $B=\operatorname{Aut}(F)$ since each automorphism of $F$ will belong to a class $B_{N}$ for some natural number $N$. In this sense it follows by the well-known result of Nielsen (see [4], [5]) that $B$ is generated by the elementary Nielsen automorphisms. If the free generators of $F$ are infinite then

$$
B<\operatorname{Aut}(F), \quad B \neq \operatorname{Aut}(F) .
$$

The following automorphism of $F_{\infty}=\left\langle x_{1}, x_{2}, \cdots\right\rangle$ shows this. Let $\beta \in \operatorname{Aut}\left(F_{\infty}\right)$ be such that $\beta\left(x_{j}\right)=x_{1} x_{2} \cdots x_{j}$ for $j=1,2, \cdots$ (then $\beta^{-1}\left(x_{j}\right)=x_{j-1}^{-1} x_{j}$ for $j=$ $2,3, \cdots$ and $\left.\beta^{-1}\left(x_{1}\right)=x_{1}\right)$. Clearly $\beta \notin B$ since $L_{x}\left(\beta\left(x_{j}\right)\right)=j$ which increases without bound.

The elementary simultaneous Nielsen automorphisms, induced by the elementary simultaneous Nielsen transformations of rank $|\gamma|$ applied on the generators $\left\{x_{\nu} \mid \nu \in \Re\right\}$, belong to the class $B_{2}$. In fact, it is obvious that $B_{1}$ consists precisely of those automorphisms which permute the generators and change their exponent signs. It can be shown (without much difficulty) that the automorphisms in $B_{2}$ may be expressed as products (compositions) of the elementary simultaneous automorphisms (and it is clear that the elementary simultaneous automorphisms are in $B_{2}$ ). As we proceed to $B_{3}$, the same result turns out to be valid but the proof (Theorem 4.6) is so heavily involved with case arguments that no easy generalization seems possible. Since we have $\left\langle B_{2}\right\rangle=\left\langle B_{3}\right\rangle$ where $B_{1} \subset B_{2} \subset B_{3} \subset \ldots$ 
$C B_{k} \subset \cdots \subset B$, it seems natural to conjecture that $\left\langle B_{2}\right\rangle=\left\langle B_{3}\right\rangle=\left\langle B_{k}\right\rangle=\langle B\rangle$ for all $k \geq 4$. This is equivalent to the conjecture that the elementary simultaneous automorphisms are a set of generators of $B$, the subgroup of bounded automorphisms.

We may easily characterize the automorphisms generated by the elementary Nielsen automorphisms, as the following theorem shows.

4.4. Theorem. Let $a \in \operatorname{Aut}(F)$ where $a\left(x_{\nu}\right)=W_{\nu}(x)$ is the freely reduced form of $\alpha\left(x_{\nu}\right)$. Then $\alpha$ is a product of elementary Nielsen automorphisms if and only if

(1) there exists some integer $N$ such that $L_{x}\left(W_{\nu}\right) \leq N$ for all $\left.\nu \in\right)$, and

(2) there exists a finite number $l$ of words $W_{\nu_{j}}\left(x_{\nu_{1}}, \cdots, x_{\nu_{l}}\right), 1 \leq j \leq l$, in the generators $\left\{x_{\nu_{1}}, \cdots, x_{\nu_{l}}\right\}$ and the remaining $W_{\nu}$ words have freely reduced expressions $A_{\nu} x_{\pi(\nu)}^{\epsilon \nu} B_{\nu}$ where $A_{\nu}, B_{\nu} \in\left\langle x_{\nu_{1}}, \cdots, x_{\nu_{l}}\right\rangle$ and $\pi$ is a permutation of the indexing set $\pi-\left\{\nu_{1}, \cdots, \nu_{l}\right\}$.

Proof. The proof that a product of elementary Nielsen transformations satisfies (1) and (2) follows by a straightforward induction on the number of factors in such a product. Conversely, if $\alpha \in \operatorname{Aut}(F)$ satisfies conditions (1) and (2), then by applying a permutation $\phi$ to the $x_{\nu}$ generators we can be sure that $a \phi: x_{\nu_{j}} \rightarrow$ $\mathbb{W}_{\nu_{j}}\left(x_{\nu_{1}}, \cdots, x_{\nu_{l}}\right)$ for $1 \leq j \leq l$, and $\alpha \phi: x_{\nu} \rightarrow A_{\nu} x_{\pi_{\phi(\nu)} \phi_{(\nu)}} B_{\nu}$ for $\nu \in \tau_{-}\left\{\nu_{1}, \cdots\right.$, $\left.\nu_{l}\right\}$. For notational convenience we replace $x_{\nu_{j}}$ by $x_{j}, 1 \leq j \leq n$, and the remaining generators $x_{\nu}$ by $x_{\mu}$, for $\mu \in \mathbb{M}$. In this notation, the automorphism $\alpha^{\prime}=\alpha \phi$ is such that

$$
\alpha^{\prime}\left(x_{j}\right)=W_{j} \in\left\langle x_{1}, \cdots, x_{l}\right\rangle \quad \text { and } \quad \alpha^{\prime}\left(x_{\mu}\right)=A_{\mu} \underset{\pi \phi(\mu)}{\epsilon_{\phi}(\mu)} B_{\mu}=W_{\phi(\mu)}
$$

where $A_{\mu}, B_{\mu} \in\left\langle x_{1}, \cdots, x_{l}\right\rangle$. By appealing to Theorem 2.2, a finite sequence of (standard) elementary Nielsen transformations can be found taking $\left(W_{1}, W_{2}, \cdots\right.$, $\left.W_{l}\right)$ into $\left(x_{1}, x_{2}, \cdots, x_{l}\right)$ while fixing the remaining words $W_{\phi(\mu)}$ (see [3]). By the structure of the words $W_{\phi(\mu)}$ and their uniform boundedness we may eliminate the initial and terminal members $A_{\mu}, B_{\mu}$ of $W_{\phi(\mu)}=A_{\mu} x_{\pi_{\phi(\mu)}{ }_{\phi(\mu)}} B_{\mu}$, for all $\mu$ by a finite application of elementary Nielsen transformations.

Thus a finite sequence $N_{\alpha_{1}}, N_{\alpha_{2}}, \cdots, N_{\alpha_{k}}$ of elementary Nielsen transformations exists such that

$$
N_{a_{k}} \cdots N_{a_{2}} N_{a_{1}}: \begin{cases}W_{j} \longrightarrow x_{j} & \text { for } 1 \leq j \leq l, \\ W_{\mu} \longrightarrow x_{\pi \phi(\mu)}^{\epsilon_{\phi(\mu)}} & \text { for } \mu \in M .\end{cases}
$$

Hence $N_{a_{k}} \cdots N_{a_{2}} N_{a_{1}} N_{a \phi}$ applied on the $x_{\nu}$ generators produces a permutation of these generators with changing exponent sign, i.e.,

$$
\left(N_{a_{k}} \cdots N_{a_{2}} N_{a_{1}} N_{a \phi}\right)\left(x_{\nu}\right)=N_{a_{k+1}}\left(x_{\nu}\right)
$$


where $a_{k+1}$ is an elementary Vielsen transformation which permutes and changes exponent signs.

By using the anti-isomorphism between the Nielsen transformations and the automorphisms of the free group $F$, we find

$$
N_{a \phi a_{1} a_{2} \cdots a_{k}}=N_{a_{k+1}}
$$

which implies that

$$
\alpha=a_{k+1} a_{k}^{-1} \cdots a_{2}^{-1} \cdot a_{1}^{-1} \phi^{-1}
$$

is a product of elementary automorphisms.

4.5. Example. We now give an example of an automorphism of $F_{\infty}$ which belongs to $B_{3}$ (i.e., is a "bounded 3" a utomorphism) but cannot be generated by the elementary Nielsen automorphisms. The automorphism $\alpha$ is defined by the mapping $a\left(x_{2 k-1}\right)=x_{2 k-1} x_{2 k+1} x_{2 k}=W_{2 k-1}, a\left(x_{2 k}\right)=x_{2 k}^{-1} x_{2 k+3} x_{2 k+2}=W_{2 k}$ for $k=1,2,3, \cdots$ whose inverse $\alpha^{-1}$ is given by $a^{-1}\left(x_{2 k-1}\right)=x_{2 k-1} x_{2 k} x_{2 k+1}^{-1}$ $=U_{2 k-1}, a^{-1}\left(x_{2 k}\right)=x_{2 k+3} x_{2 k+2}^{-1} x_{2 k}^{-1}=U_{2 k}$ for $k=1,2,3, \cdots$. Note that $\alpha$ is also a triangular automorphism. We show that condition (2) of Theorem 4.4 is not satisfied by this automorphism. Suppose on the contrary that criterion (2) holds here, then we can find $l$ words $w_{\nu_{1}}\left(x_{\nu_{1}}, \cdots, x_{\nu_{l}}\right), \cdots, w_{\nu_{l}}\left(x_{\nu_{1}}, \cdots, x_{\nu_{l}}\right)$ in the $l$ generators $x_{\nu_{1}}, \cdots, x_{\nu_{l}}$. We may assume that $x_{\nu_{1}}, \cdots, x_{\nu_{l}}$ have been already arranged so that $x_{\nu_{j}}<x_{\nu_{j+1}}$ for $j=1,2, \cdots, l-1$ and that $W_{\nu_{j}}=W\left(x_{\nu_{j}}\right)$ for $j=1,2, \cdots, l$ (we are using the notation in Theorem 3.3). It then follows that $x_{\nu_{l}}$ is the "latest" occurring $x$ generator in the expressions $w_{\nu_{1}}, \cdots, W_{\nu_{l}}$ ' and in particular we would have that $W_{\nu_{l}}$ is expressible only in terms of $x_{\nu_{l}}$, i.e., $W_{\nu_{l}}=x_{\nu_{l}}^{\epsilon}, \epsilon= \pm 1$. This contradicts the definition of the automorphism $\alpha$. Thus $a$ does not satisfy condition (2).

We shall later show that the automorphism defined above is generated by the elementary simultaneous Nielsen automorphisms. It is now clear that the subgroup generated by the elementary simultaneous automorphisms strictly contains the subgroup generated by the elementary automorphisms.

We now enter into the proof of the following proposition.

4.6. Theorem. Let $B_{3}$ be the class of all "bounded 3" automorphisms of $F$, the free group on the free generators $\left\{x_{\nu} \mid \nu \in \eta\right\}$. Then the group generated by $B_{3}$ is identical to the group generated by the elementary simultaneous Nielsen transformations (a fortiori $\left\langle B_{2}\right\rangle=\left\langle B_{3}\right\rangle$ ).

We shall show that if $\alpha \in B_{3}$ then $\alpha$ is a product of elementary simultaneous Nielsen automorphisms. To produce this result, we shall express each $x$ generator of $\left.\left\{x_{\nu} \mid \nu \in\right)\right\}$ as a freely reduced word in the "W" generators, where $a\left(x_{\nu}\right)=W_{\nu}$ 
is freely reduced in the $x$ generators, e.g., $x=\Pi_{j=1}^{k} W_{j}{ }_{j}$ and $k \leq 3, \epsilon_{j}= \pm 1$. We shall then apply certain elementary simultaneous Nielsen transformations on the system $\left\{x_{\nu} \mid \nu \in \Re\right\}$ to obtain a system of generators which will be Nielsen reduced (see [3]) when viewed as words in the $W$ generators. It shall then follow that the resulting system of generators is $\left\{w_{\pi(\nu)}^{\epsilon} \mid \epsilon_{\nu}= \pm 1, \nu \in \Re\right\}$ where $\pi: \Re \rightarrow \pi$ is a permutation of the set $\Re$. By applying a permutation with changing exponent sign on the resulting system, we shall succeed in converting $\left\{x_{\nu} \mid \nu \in \Re\right\}$ to $\left\{W_{\nu} \mid \nu \in \mathcal{K}\right\}$ by a finite application of elementary simultaneous Nielsen transformations. By using the anti-isomorphism of $\operatorname{Aut}(F)$ onto $N(F)$, the group of Nielsen transformations of $F$, we will have shown that $\alpha \in B_{3}$ is a finite product of elementary simultaneous automorphisms.

Canonical forms for the $x_{\nu}$ generators. By replacing $x_{\nu}$ by its inverse $x_{\nu}^{-1}$, if necessary, and calling the resulting generator $\xi_{\nu}$ we can be sure that $\xi_{\nu}$ falls in one and only one of the following categories:

1. $\xi_{\nu}=A B C$ where $A, B, C \in\left\{W_{\nu}\right\} \cup\left\{W_{\nu}^{-1}\right\}$, the expression being freely reduced as a word in the $W$ generators and

$$
\begin{aligned}
L_{x}(A) & =L_{x}(B)=L_{x}(C)=3, \\
L_{x}(A B) & =4, \quad L_{x}(B C)=2 .
\end{aligned}
$$

2. $\xi_{\nu}=A a B$ where $A, a, B \in\left\{W_{\nu}\right\} \cup\left\{W_{\nu}^{-1}\right\}$,

$$
\begin{aligned}
L_{x}(A) & =L_{x}(B)=3, \quad L_{x}(a)=1 ; \quad \text { and } \\
L_{x}(A a) & =4, \quad L_{x}(a B)=2 .
\end{aligned}
$$

3. $\xi_{\nu}=A B a$ where $A, B, a \in\left\{W_{\nu}\right\} \cup\left\{W_{\nu}^{-1}\right\}$ and

$$
\begin{aligned}
L_{x}(A) & =L_{x}(B)=3, \quad L_{x}(a)=1, \\
L_{x}(A B) & =2, \quad L_{x}(B a)=2 .
\end{aligned}
$$

4. $\xi_{\nu}=A a b$ where $A, a, b \in\left\{W_{\nu}\right\} \cup\left\{W_{\nu}^{-1}\right\}$ and

$$
L_{x}(A)=3, \quad L_{x}(a)=L_{x}(b)=1 \text {. }
$$

5. $\xi_{\nu}=a A b$ where $A, a, b \in\left\{W_{\nu}\right\} \cup\left\{W_{\nu}^{-1}\right\}$ and

$$
\begin{aligned}
& L_{x}(a)=L_{x}(b)=1, \\
& L_{x}(A)=3, \quad L_{x}(a A)=L_{x}(A b)=2 .
\end{aligned}
$$

6. $\xi_{\nu}=T A \tau$ where $T, A, \tau \in\left\{W_{\nu}\right\} \cup\left\{W_{\nu}^{-1}\right\}$ and

$$
\begin{aligned}
L_{x}(T) & =L_{x}(\tau)=2, \quad L_{x}(A)=3, \quad \text { also } \\
L_{x}(T A) & =3, \quad L_{x}(A \tau)=1 .
\end{aligned}
$$


7. $\xi_{\nu}=T A \tau$ where $T, A, \tau \in\left\{W_{\nu}\right\} \cup\left\{W_{\nu}^{-1}\right\}$ and

$$
\begin{aligned}
L_{x}(T) & =L_{x}(\tau)=2, \quad L_{x}(A)=3, \\
L_{x}(T A) & =L_{x}(A \tau)=1 .
\end{aligned}
$$

8. $\xi_{\nu}=T \tau A$ where $T, A, \tau \in\left\{W_{\nu}\right\} \cup\left\{W_{\nu}^{-1}\right\}$ and

$$
\begin{aligned}
& L_{x}(T)=L_{x}(\tau)=2, \quad L_{x}(A)=3, \quad \text { also } \\
& L_{x}(T r)=4, \quad L_{x}(\tau A)=1 .
\end{aligned}
$$

9. $\xi_{\nu}=A \operatorname{Tr}$ where $A, T, \tau \in\left\{W_{\nu}\right\} \cup\left\{W_{\nu}^{-1}\right\}$ and

$$
\begin{aligned}
L_{x}(T) & =L_{x}(r)=2, \quad L_{x}(T \tau)=2, \quad \text { also } \\
L_{x}(A T) & =3, \quad L_{x}(A)=3 .
\end{aligned}
$$

10. $\xi_{\nu}=T \pi g$ where $T, \tau, g \in\left\{W_{\nu}\right\} \cup\left\{W_{\nu}^{-1}\right\}$ and

$$
\begin{aligned}
& L_{x}(T)=L_{x}(\tau)=2, \quad L_{x}(T \tau)=2, \quad \text { also } \\
& L_{x}(g)=1, \quad L_{x}(\tau g)=1 .
\end{aligned}
$$

11. $\xi_{\nu}=T g \tau$ where $T, g, \tau \in\left\{W_{\nu}\right\} \cup\left\{W_{\nu}^{-1}\right\}$ and

$$
\begin{aligned}
L_{x}(T) & =L_{x}(\tau)=2, \quad L_{x}(g)=1, \quad \text { also } \\
L_{x}(T g) & =3, \quad L_{x}(g \tau)=1 .
\end{aligned}
$$

12. $\xi_{\nu}=B S$ where $B, S \in\left\{W_{\nu}\right\} \cup\left\{W_{\nu}^{-1}\right\}$ and

$$
L_{x}(B)=3, \quad L_{x}(S)=2 .
$$

13. $\xi_{\nu}=T \theta$ where $T, \theta \in\left\{W_{\nu}\right\} \cup\left\{W_{\nu}^{-1}\right\}$ and

$$
L_{x}(T)=2, \quad L_{x}(\theta)=1 .
$$

14. $\xi_{\nu}=W$ where $W \in\left\{W_{\nu}\right\}$ and

$$
\left.L_{x}(W)=1 \quad \text { (i.e., } W=x_{\nu}^{ \pm 1}\right) \text {. }
$$

Let $E_{j}$ denote the collection of all $\xi_{\nu}$ such that $\xi_{\nu}$ satisfies condition $j$, for $j=1,2, \cdots, 14$. By a quick check, it can be seen that each $x_{\nu}$ generator may be put into its canonical form in just one way, so that $\bigcup_{j=1}^{14} E_{j}$ is a free set of generators of $F$. We note that $\bigcup_{j=1}^{9} E_{j}$ consists of those $x_{\nu}$ or their inverses with " $W$ " length 3 which contain at least one $W$ symbol of $x$ length 3 . $E_{10} \cup E_{11}$ consists of those $x_{\nu}$ generators (or their inverses) which have $W$ length 3 but which have no $W$ symbol of $x$ length $3 . E_{12} \cup E_{13}$ consists of those $x_{\nu}$ generators (or their inverses) with $W$ length $2 . E_{14}$ consists of those $x_{\nu}$ generators 
(or their inverses) which are already $W$ symbols.

We now work with the set $\bigcup_{j=6}^{14} E_{j}$, viewing its elements as words in the $W$ generators; we will Nielsen reduce this set with respect to itself by elementary simultaneous transformations so that the resulting system of $W$ words is Nielser. reduced (with respect to itself).

The following lemmas are stated without proof and may be checked by routine arguments involving simultaneous use of the $x$-length and $W$-length of the generators $\left\{\xi_{\nu} \mid \nu \in \Re\right\}$.

4.7. Lemma. By application of a finite number of elementary simultaneous Nielsen transformations, $E_{j}$ may be converted to $\bar{E}_{j}$, for $6 \leq j \leq 14, j \neq 9$, where $\bar{E}_{j}$ consists of the set of W initial elements of $\xi_{\nu} \in E_{j}$ when expressed in terms of the W symbols.

4.8. Lemma. The set $E_{9}$ may be converted to the set $\bar{E}_{9}$ of $W$ initial elements of $\xi_{\nu} \in E_{9}$ by application of elements from $\bigcup_{j=6 ; j \neq 9}^{13} \bar{E}_{j}$ to $E_{9}$ using simultaneous Nielsen transformations.

We now proceed to alter the sets $E_{j}, j=1,2,3,4,5$, in such a manner that after transformation, the set $E_{3}$ will be converted to a set of $W$ symbols of $x$ length 3.

Step I. Apply elements from $\bar{E}_{14}$ so as to convert $E_{3}$ to $\bar{E}_{3}$ consisting of the major initial segments of the words in $E_{3}$. Similarly convert $E_{4}$ to $\bar{E}_{4}$ consisting of the initial elements (which are $W$ symbols) of the words in $E_{4}$ by using elements from $\bar{E}_{14}$; convert $E_{5}$ to $\bar{E}_{5}$, consisting of the central elements (which are $W$ symbols) of the words in $E_{5}$.

Step II. Collect into distinct classes those words in $E_{1}$ which share the same terminal segments. To those classes whose major terminal segments are elements in $\bar{E}_{3} \cup \bar{E}_{3}^{-1}$ apply the appropriate elements in $\bar{E}_{3}$ to reduce such classes to classes consisting of $W$ symbols of $x$ length 3 . Denote this transformed set by $\bar{E}_{1}$. ( $\bar{E}_{1}$ may well still contain words in the $W$ symbols of $W$ length 3.$)$

Step III. In $E_{2}$, collect into disjoint subsets those words in $E_{2}$ whose terminal elements are the same $W$ symbol (or its inverse) which belongs to $\bar{E}_{4}$. Reduce these words by the proper application of elements in $\bar{E}_{4} \cup \bar{E}_{14}$ to convert these subsets to $W$ symbols of $x$ length 3 . (There may well be elements in $E_{2}$ which have been unaffected by these transformations.)

Let $\bar{E}_{2}^{\prime}$ be those words in $E_{2}$ unaffected in this step. Thus $E_{2}$ is converted to $\bar{E}_{2} \cup \bar{E}_{2}^{\prime}$ where $\bar{E}_{2}$ is the transformed subset of $E_{2}$.

Step IV. Collect those words in $\bar{E}_{3}$ whose right halves are $W$ symbols in $\bar{E}_{5} \cup \bar{E}_{5}^{-1}$; collect them into classes and reduce them by proper application of $W$ symbols in $\bar{E}_{5}$ so as to produce a collection of $W$ symbols; call it $\bar{E}_{3}^{\prime}$. The remaining unaffected words in $\bar{E}_{3}$ will be called $\bar{E}_{3}^{*}$. 
Step V. We will now convert $\bar{E}_{3}^{*}$ into a collection of $W$ symbols of $x$ length 3 . For this purpose we require the following:

4.9. Lemma. Let $\xi=A B a \in E_{3}$ be such that $A B \in \bar{E}_{3}^{*}$, where $A, B, a \in\left\{W_{\nu}\right\} \cup$ $\left\{W_{\nu}^{-1}\right\}$. Then $B^{ \pm 1} \in \bar{E}_{1} \cup \bar{E}_{2} \cup \bar{E}_{3}^{\prime} \cup \bar{E}_{4} \cup \bar{E}_{9} \cup \bar{E}_{12}$.

The verification is similar to the previous combinatorial lemmas and is omitted.

Now by collecting into separate subsets those words in $\bar{E}_{3}^{*}$ whose right halves belong to $\bar{E}_{1} \cup \bar{E}_{1}^{-1}$, those whose right halves belong to $\bar{E}_{2} \cup \bar{E}_{2}^{-1}$, etc., and applying the appropriate $W$ symbols ( $x$ length 3 ) in $\bar{E}_{1} \cup \bar{E}_{2} \cup \ldots \cup \bar{E}_{12}$ we may reduce $\bar{E}_{3}^{*}$ to the set of left halves of words in $\bar{E}_{3}^{*}$. These $W$ symbols are of $x$ length 3. Call this set $\bar{E}_{3}^{* *}$. We have therefore converted $\bar{E}_{3}$ into $\bar{E}_{3}^{\prime} \cup \bar{E}_{3}^{* *}$, a set consisting of the initial elements of the words in $E_{3}$. Set $\widetilde{E}_{3}=\bar{E}_{3}^{\prime} \cup \bar{E}_{3}^{* *}$, then $\widetilde{E}_{3}$ consists of all $W$ initial elements of $\xi_{\nu} \in E_{3}$.

Up to this point we have converted $E_{3}$ into $\tilde{E}_{3}$, and $E_{j}$ into $\bar{E}_{j}$ for $j=4,5$, $\cdots, 14$. These new collections each consist of $W$ symbols. We may also reduce $\bar{E}_{2}^{\prime}$ to the same type: a set of $W$ symbols of $x$ length 3 by using a finite sequence of elementary simultaneous Nielsen transformations and using the $W$ symbols in $\bar{E}_{1} \cup \bar{E}_{2} \cup \widetilde{E}_{3} \cup \bar{E}_{4} \cup \bar{E}_{5} \cup \bar{E}_{9} \cup \bar{E}_{12}$.

This follows by appealing to the following lemma.

4.10. Lemma. Let $\xi=A a B \in \bar{E}_{2}^{\prime}$, then $B^{ \pm 1} \in \bar{E}_{1} \cup \bar{E}_{4} \cup \widetilde{E}_{3} \cup \bar{E}_{4} \cup \bar{E}_{5} \cup \bar{E}_{9} \cup \bar{E}_{12}$.

We may thus collect the elements in $\bar{E}_{2}^{\prime}$ of $W$ length 3 in the following manner

Put in the first collection those words of $\bar{E}_{2}^{\prime}$ whose terminal elements are $W$ symbols in $\bar{E}_{1}$; in the second collection those words of $\bar{E}_{2}^{\prime}$ whose terminal elements are $W$ symbols in $\widetilde{E}_{2}$; etc., $\cdots$. In.each such collection we may apply an elementary simultaneous transformation using the appropriate elements in $\bar{E}_{1} \cup$ $\widetilde{E}_{3} \cup \bar{E}_{4} \cup \cdots \cup \bar{E}_{14}$. Next, apply the appropriate elements in $\bar{E}_{14}$ to produce the desired set of $W$ symbols. The set which results along with $\bar{E}_{2}$ will be denoted by $\widetilde{E}_{2}$. The set $\tilde{E}_{2}$ consists of the initial elements of the words in $E_{2}$.

We return now to the set $\bar{E}_{1}$ and convert it to a set of $W$ symbols of $x$ length 3 by using a finite succession of elementary simultaneous Nielsen transformations.

We collect into distinct classes those words in $\bar{E}_{1}$ which share the same major terminal segments. To put things into focus, let us denote such a class by

$$
\left\{\begin{array}{l}
\xi=A B C \\
\xi_{\nu}=A_{\nu} B C \\
\cdot .
\end{array}\right.
$$

In each such class pick a representative $\xi=A B C$, for example. (Of course, such a representative might already be a $W$ symbol, $A_{\nu}$ as might be the case.) In each such class apply the representative to the remaining words in its class to produce the set 


$$
\left\{\begin{array}{c}
\xi=A B C \\
\xi_{\nu} \xi^{-1}=A_{\nu} A^{-1} \\
\cdot
\end{array}\right.
$$

We now consider a typical representative: $\xi=A B C$. Let $A=\xi g b$ be the reduced $x$ expression for $A$, so that $g=C^{-1} B^{-1} b^{-1}$. Now the following situations may occur:

(i) $L_{w}(b)=1$. Then $g=C^{-1} B^{-1} b^{-1} \in E_{3}$ and thus $A \in \bar{E}_{1}$ (recall the construction of $\bar{E}_{1}$ ) so that $\xi=A B C$ has already been converted to $A$ in $\bar{E}_{1}$.

(ii) $L_{w}(b)=2$. I.et $b=P Q$ be its $W$ expression. Then $g=C^{-1} B^{-1} Q^{-1} P^{-1}$ so that $B=Q^{-1}$ and thus $b^{-1}=B P^{-1}, g=C^{-1} P^{-1}$ implying that $B, C^{-1} \in \bar{E}_{12}$. (Clearly, $b \neq g$ since if not $\Rightarrow B=P$ so that $b^{-1} \approx 1$; also it is obvious that $b^{-1} \neq g$ since $L_{w}(A)=3$.)

(iii) $L_{w}(b)=3$. Set $b=P Q R$ where $P, Q, R \in\left\{W_{\nu}\right\} \cup\left\{W_{\nu}^{-1}\right\}$. Then $g=$ $C^{-1} B^{-1} R^{-1} Q^{-1} P^{-1} \Rightarrow R=B^{-1}$ and thus

$$
b^{-1}=B Q^{-1} P^{-1}, \quad g=C^{-1} Q^{-1} P^{-1} .
$$

Now in this case (iii), $b$ will fall in one and only one of the following categories:

(a) $b^{ \pm 1} \in E_{2}$. If $L_{x}\left(Q^{-1} P^{-1}\right)=4$, then from the expressions in (*) above it follows that $b^{-1}=g$, since $L_{x}(B)=L_{x}\left(C^{-1}\right)=3$, which is impossible. Thus $L_{x}\left(Q^{-1} P^{-1}\right)=2$, so that $b^{-1}, g \in E_{2}$ and $B \in \widetilde{E}_{2}$ and $C^{-1} \in \widetilde{E}_{2}$.

(b) $b^{-1} \in E_{3}$. Then $g \in E_{3}$ so that $B, C^{-1} \in \widetilde{E}_{3}$.

(c) $b^{-1} \in E_{4}$. Then $g \in E_{4}$ so that $B, C^{-1} \in \bar{E}_{4}$.

(d) $b^{-1} \in E_{9}$. Then $g \in E_{9}$ so that $B, C^{-1} \in \bar{E}_{9}$. (Note that $b^{ \pm 1} \notin E_{\text {s }}$ since $L_{x}(B)=3$; also $b \notin E_{8}$ for the same reason.)

(e) $b^{-1} \in E_{1}$. So that $g \in E_{1}$ and $b^{-1}=B Q^{-1} P^{-1}, g=C^{-1} Q^{-1} P^{-1}$.

Let us call those representatives that satisfy the conditions in (i), (ii) or (iii) (a)-(d), above, representatives which are of category I. Also call those representatives which satisfy condition (iii) (e) representatives of category II.

By applying the $W$ symbols from $\widetilde{E}_{2} \cup \widetilde{E}_{3} \cup \bar{E}_{4} \cup \bar{E}_{9} \cup \bar{E}_{12}$ to those representatives which satisfy category I we may convert the representatives $\{\xi=A B C\}$ by elementary simultaneous transformations to $\{A B\}$, then further to $\{A\}$. Thus for those classes whose representatives satisfy category I we have succeeded in transforming them to

$$
\left\{\begin{array}{c}
A \\
\xi_{\nu} \xi^{-1}=A_{\nu} A^{-1}
\end{array}\right.
$$

Using an elementary simultaneous Nielsen transformation, we can convert the classes 


$$
\left\{\begin{array} { c } 
{ A } \\
{ A _ { \nu } A ^ { - 1 } } \\
{ \cdot . }
\end{array} \text { to } \left\{\begin{array}{l}
A \\
A_{\nu} \\
\cdot .
\end{array}\right.\right.
$$

so that those classes whose representatives are in cateogry I can be converted to $W$ symbols of $x$ length 3 .

For those representatives which satisfy category II above, e.g.,

$$
\left\{\begin{array}{c}
\xi=A B C \\
\xi_{\nu} \xi^{-1}=A_{\nu} A^{-1} \\
\cdot \quad \cdot
\end{array}\right.
$$

we know that $b^{-1}=B Q^{-1} P^{-1}, g=C^{-1} Q^{-1} P^{-1}$ as in (iii) (e). Let $\zeta=K Q^{-1} P^{-1}$ be the representative of the class containing $b^{-1}, g$ ( $\zeta$ might be $b^{-1}$ or $g$, in which case we argue similarly). Thus the class appears as

$$
\left\{\begin{array}{c}
\zeta=K Q^{-1} P^{-1} \\
b^{-1}=B Q^{-1} P^{-1} \\
g=C^{-1} Q^{-1} P^{-1} \\
\cdot \cdot \cdot \cdot
\end{array}\right.
$$

which already has been transformed to

$$
\left\{\begin{array}{rl}
\zeta & =K Q^{-1} P^{-1} \\
b^{-1} \zeta^{-1} & =B K^{-1} \\
g \zeta^{-1} & =C^{-1} K^{-1} \\
\cdot & \cdot
\end{array} .\right.
$$

We apply an elementary simultaneous transformation which converts the category II representatives $\xi=A B C$ to $(A B C) C^{-1} K^{-1}=A B K^{-1}$, then apply the elementary simultaneous transformation which converts $A B K^{-1}$ to $\left(A B K^{-1}\right)\left(B K^{-1}\right)^{-1}=$ $A$ for each "new" representative.

The category II classes have now been converted to

$$
\left\{\begin{array}{c}
A \\
A_{\nu} A^{-1} \\
\cdot .
\end{array}\right.
$$

We finally apply the elementary simultaneous transformation which converts these classes 


$$
\left\{\begin{array} { c } 
{ A } \\
{ A _ { \nu } A ^ { - 1 } } \\
{ \cdot . }
\end{array} \text { to } \left\{\begin{array}{l}
A \\
A_{\nu} \\
\cdot .
\end{array}\right.\right.
$$

The union of the resulting classes whose "ancestors" were in categories I and II will be called $\widetilde{E}_{1}$. $\widetilde{E}_{1}$ consists purely of initial W symbols with $x$ length 3 which belong to $E_{1}$.

Hence having applied a finite number of elementary Nielsen transformations (finite but tediously large in number) we have succeeded in converting the $\left\{\xi_{\nu}\right\}$ generators $\left\{\xi_{\nu}\right\}=\bigcup_{j=1}^{14} E_{j}$ into the set $\widetilde{E}_{1} \cup \widetilde{E}_{2} \cup \widetilde{E}_{3} \cup\left(\bigcup_{j=4}^{14} \bar{E}_{j}\right)$ consisting of all the $\left\{W_{\nu}\right\}$ symbols save for exponent sign which are the initial $W$ elements of the canonical forms $\xi_{\nu} \in \bigcup_{j=1}^{14} E_{j}$.

This completes the proof of Theorem 4.6.

Applying the process described in the proof of Theorem 4.6 to the specific "bounded 3" automorphism described in Example 4.5 we find that $a=\alpha_{1} \alpha_{2} \alpha_{3} \alpha_{4}$ where

$$
\begin{aligned}
& a_{1}\left(x_{2 k-1}\right)=x_{2 k-1}, a_{1}\left(x_{2 k}\right)=x_{2 k}^{-1} \text { for } k=1,2,3, \ldots ; \\
& a_{2}\left(x_{1}\right)=x_{1}, a_{2}\left(x_{2 k}\right)=x_{2 k}, a_{2}\left(x_{2 k+1}\right)=x_{2 k+1} x_{2 k}^{-1} \text { for } k=1,2,3, \ldots ; \\
& a_{3}\left(x_{1}\right)=x_{1} x_{3}, a_{3}\left(x_{2 k-2}\right)=x_{2 k-2} x_{2 k+1}, a_{3}\left(x_{2 k-1}\right)=x_{2 k-1} \quad \text { for } k=2,3,4, \ldots ; \\
& a_{4}\left(x_{1}\right)=x_{1}, a_{4}\left(x_{2 k}\right)=x_{2 k}, a_{4}\left(x_{2 k+1}\right)=x_{2 k+1} x_{2 k} \text { for } k=1,2,3, \ldots
\end{aligned}
$$

are the required simultaneous transformations.

5. Bounded "two occurring" automorphisms of $F$. In this section we study a special class $T$ of $\operatorname{Aut}(F)$ as defined below and show that the class $T \cap B$ is contained in the subgroup of $\operatorname{Aut}(F)$ generated by the elementary simultaneous Nielsen automorphisms.

Let $F$ be the free group generated by the free generators $\left\{x_{\nu} \mid \nu \in \Re\right\}$. Suppose $U \in F$ is a freely reduced word in the $x_{\nu}$ generators.

5.1. Definition. The function $\sigma_{x}: F \rightarrow Z_{+}$is defined as follows: $\sigma_{x}(U)$ is the number of distinct $x_{\nu}$ generating symbols occurring in $U$.

5.2. Definition. An automorphism $a: x_{\nu} \rightarrow W_{\nu}$, where $W_{\nu}$ is freely reduced in the $x$ generators, is said to be of class $T$ if and only if $\sigma_{x}\left(W_{\nu}\right) \leq 2$ for all $\nu \in \Re$. (Note that $T$ is not a group since $a \in T$ does not guarantee that $\alpha^{-1} \in T$.)

We now state the following theorem.

5.3. Theorem. Let $a \in T$, where $a\left(x_{\nu}\right)=W_{\nu}$ is the freely reduced form of $\alpha\left(x_{\nu}\right)$, for all $\nu \in \Re$. Let $x_{\nu_{1}} \in\left\{x_{\nu}\right\}$ be fixed and let $\left\{w_{\nu_{1}}, w_{\nu_{2}}, \cdots, w_{\nu_{r}}\right\}$ be the smallest subset of $W_{\nu}$ generators required to generate $x_{\nu} \cdot$ 
Then the number of distinct $x$ generators occurring among the words $W_{\nu_{1}}$, $W_{\nu_{2}}, \cdots, W_{\nu_{r}}$ is $r$. Denote these generators by $x_{\nu_{1}}, x_{\nu_{2}}, \cdots, x_{\nu_{r}}$. Then $\left\langle x_{\nu_{1}}\right.$, $\left.x_{\nu_{2}}, \cdots, x_{\nu_{r}}\right\rangle^{\prime}=\left\langle W_{\nu_{1}}, W_{\nu_{2}}, \cdots, w_{\nu_{r}}\right\rangle$.

In order to facilitate the proof of Theorem 5.3, we need the next two lemmas. The groups which we consider in these lemmas will be assumed torsion free.

5.4. Lemma. Let $G=A *\langle V\rangle$ (free product of group $A$ with cyclic group $\langle V\rangle$ ). If $a_{1}, a_{2} \in A$, then $\left\langle A, a_{1} V a_{2}\right\rangle=A *\left\langle a_{1} V a_{2}\right\rangle$.

The proof follows by appealing to a standard characterization property of a free product by homomorphic extensions (see e.g., [3]).

We proceed with the next lemma.

5.5. Lemma. Let $G=A * B$, where $A, B \neq 1$. Let $U_{1}, \cdots, U_{k} \in A, V \in A * B$ and $V \notin A$. If an element of $A$ can be expressed as a word in the symbols $U_{1}$, $\cdots, U_{k}, V$ then it can also be expressed without the use of $V$, i.e., if $W\left(U_{1}, \cdots, U_{k}, V\right) \in A$ then $W\left(U_{1}, \cdots, U_{k}, V\right)=R\left(U_{1}, \cdots, U_{k}\right)$.

Proof. Let $g \in A$ and suppose $g=W\left(U_{1}, \cdots, U_{k}, V\right)$ is an expression for this element as a word in the symbols $U_{1}, \cdots, U_{k}, V$. Express $V$ in the normal form of a free product $V=a_{1} b_{1} a_{2} b_{2} \cdots b_{2} a_{s+1}$ where $a_{j} \in A, b_{j} \in B$ and $a_{j}$, $b_{j} \neq 1$ for $j=2, \cdots, s$ except for $a_{1}$ or $a_{s+1}$. Set $\bar{V}=b_{1} a_{2} b_{2} \cdots a_{s} b_{s}$.

Clearly, $\langle A, \bar{V}\rangle=A *\langle\bar{V}\rangle$ so that by Lemma 5.4 we have $\left\langle A, a_{1} \bar{V} a_{s+1}\right\rangle=$ $A *\left\langle a_{1} \bar{V} a_{s+1}\right\rangle$, i.e., $\langle A, V\rangle=A *\langle V\rangle$.

Choose an expression for $g$ of smallest word length in the symbols $U_{1}, \cdots$, $U_{k}, V$ and assume that the $V$ symbol occurs in this expression. This expression for $g$ may be written as

$$
g=A_{1}\left(U_{j}\right) V^{n}{ }^{n} A_{2}\left(U_{j}\right) V^{n}{ }^{n} \ldots A_{k}\left(U_{j}\right) V^{n}{ }^{n} A_{k+1}\left(U_{j}\right)
$$

where $A_{l}\left(U_{j}\right)$ are the expressions in the $U_{j}$ symbols grouped together $\left(A_{l} \neq 1\right.$ for $l=2, \cdots, k$ by virtue of the minimal length). But then $V^{n_{1}} A_{2} V^{n_{2}} \cdots V^{n_{k}}$ and $A_{1}^{-1} g A_{k+1}^{-1}$ are two different reduced sequences for the same element in the free product $A *\langle V\rangle$. Thus the $V$ symbol does not occur in an expression for $g$ of minimum length in the symbols $U_{1}, \cdots, U_{k}, V$.

To avoid notational inconvenience in the proof of Theorem 5.3 we relabel $x_{\nu_{1}}$ as $x_{1}, W_{\nu_{1}}$ as $W_{1}$ etc., in the statement of the theorem.

Proof of Theorem 5.3. Consider the set $\left\{W_{1}, W_{2}, \cdots, w_{r}\right\}$, then $x_{1}$ is a word in these $W$ symbols, i.e., $x_{1}=R\left(W_{1}, \cdots, w_{r}\right)$. At least one of these $W$ symbols contains $x_{1}$; call it $W_{1}$. If $W_{1}$ contains no other $x$ symbol then $W_{1}=x_{1}^{ \pm 1}$ and $r=1$.

Suppose that $W_{1}=W_{1}\left(x_{1}, x_{2}\right), W_{2}=W_{2}\left(x_{2}, x_{3}\right), \cdots, W_{j}=W_{j}\left(x_{j}, x_{j+1}\right)$ where 
$j<r$ and $x_{k} \neq x_{l}$ if $k \neq l \leq j+1$ (i.e., $\sigma_{x}\left(W_{k}\right)=2$ for $k=1,2, \cdots, j$ ). If no other $W$ symbol (aside from $W_{j}$ ) in $\left\{W_{1}, \cdots, W_{j}, \cdots, W_{r}\right\}$ contains $x_{j+1}$, then let

$$
\begin{aligned}
& A=\left\langle x_{\nu} \mid x_{\nu} \neq x_{j+1}, \nu \in \Re\right\rangle, \\
& B=\left\langle x_{j+1}\right\rangle, \text { so that } F=A * B .
\end{aligned}
$$

Thus, $W_{j} \notin A$ while

$$
w_{1}, \cdots, w_{j-1}, w_{j+1}, \cdots, w_{r} \in A \text { and } x_{1}=R\left(W_{1}, \cdots, w_{j-1}, w_{j}, W_{j+1}, \cdots, W_{r}\right) \in A \text {, }
$$

so that the hypotheses of Lemma 5.5 are satisfied. Therefore $x_{1}$ may be expressed without the use of $W_{j}$, contradicting the minimality of $r$. Therefore, if $j<r$, we may find a $W$ symbol, say $W_{j+1}$, which also contains $x_{j+1}$.

We have shown that we may produce the sequence $W_{1}\left(x_{1}, x_{2}\right), W_{2}\left(x_{2}, x_{3}\right), \cdots$, $W_{r-1}\left(x_{r-1}, x_{r}\right), W_{r}\left(x_{r}, x_{l}\right)$ where $W_{r}\left(x_{r}, x_{l}\right)$ is a $W$ symbol involving $x_{r}$ and $x_{l}$ where $l$ is some number $\leq r$. It therefore follows that the number of distinct occurring $x$ generators in the symbols $W_{1}, \cdots, W_{r}$ is indeed $r$.

By using Theorem 2.2 we obtain $\left\langle x_{1}, \cdots, x_{r}\right\rangle=\left\langle W_{1}, \cdots, W_{r}\right\rangle$.

We now link up the class $T \cap B$ with the elementary simultaneous Nielsen transformations.

5.6. Theorem. The group generated by the class $T \cap B$, i.e., $\langle T \cap B\rangle$ is the same as the group generated by the elementary simultaneous Nielsen automorphisms.

Proof. It is clear that each elementary simultaneous Nielsen automorphism belongs to the class $T \cap B$. It will be sufficient to show that if $\alpha \in T \cap B$ then a can be expressed as a product of elementary simultaneous automorphisms in order to complete the proof. This is taken care of by the following lemma.

5.7. Lemma. Let $F$ be the free group on the free generators $\left\{x_{\nu} \mid \nu \in \Re\right\}$. Let $a: x_{\nu} \rightarrow W_{\nu}$ be an automorphism in class $T$ such that for some integer $N$, $L_{x}\left(\alpha^{\epsilon}\left(x_{\nu}\right)\right) \leq N$, for $\epsilon= \pm 1$ and all $\nu \in \Re$. Then there is a free product decomposition of $F={ }^{*} \Pi_{j \leq a} X_{j}, \tau$ being some ordinal, and there exists a permutation $\pi$ of the set $\left\{x_{\nu}\right\}$ such that $\left\langle a \pi\left(X_{j}\right)\right\rangle=\left\langle X_{j}\right\rangle$. There is an integer $\bar{N}$ such that the automorphism $a \pi$ is a product of $\bar{N}$ elementary simultaneous Whitebead automorpbisms where $\bar{N}<N^{2}+1$.

Proof. Let $x_{1} \in\left\{x_{\nu}\right\}$. By Theorem 5.3, we can find $W_{1}, w_{2}, \cdots, w_{r}$ such that $\left\langle x_{1}, x_{2}, \cdots, x_{r}\right\rangle=\left\langle W_{1}, W_{2}, \cdots, W_{r}\right\rangle_{\text {. It }}$ It clear that $r \leq N$ since $L_{x}\left(\alpha^{-1}\left(x_{1}\right)\right) \leq N$ implies that number of distinct $W$ symbols occurring in the freely reduced $W$ word representing $x_{1}$ is no larger than $N$.

Let $H_{x_{j}}=\left\{W_{\nu} \mid W_{\nu} \notin\left\{W_{1}, \cdots, W_{r}\right\}\right.$ and $W_{\nu}$ contains $\left.x_{j}^{\epsilon}, \epsilon= \pm 1\right\}$ for $j=1,2$, $\cdots, r$. Note that the sets $H_{x_{1}}, H_{x_{2}}, \cdots, H_{x_{r}}$ are pairwise disjoint since if $W \in H_{x_{j}} \cap H_{x_{k}}$ then $W=W\left(x_{j}, x_{k}\right)$ where $j, k \leq r$; but then $\left\langle W_{1}, W_{2}, \cdots, W_{r}, W\right\rangle=$ 
$\left\langle x_{1}, x_{2}, \cdots, x_{r}\right\rangle$ so that the symbols $W_{1}, W_{2}, \cdots, W_{r}, W$ are not free (contradiction). Therefore, $H_{x_{j}} \cap H_{x_{k}}=\varnothing$ for $1 \leq j \neq k \leq r$.

Let $W, U \in H_{x_{j}}$ where $W, U$ are distinct symbols, say $W=W\left(\theta, x_{j}\right)$, and $U=$ $U\left(\phi, x_{j}\right)$. We show that $\theta \neq \phi$, i.e., the symbols have different $x$ generators. Thus, suppose $\theta=\phi$, then it follows that $\langle W, U\rangle=\left\langle\theta, x_{j}\right\rangle$ (by Theorem 2.2) so that $x_{j} \in\langle W, U\rangle$ while $x_{j} \in\left\langle W_{1}, \cdots, W_{r}\right\rangle$; this contradicts the unique expression for $x_{j}$ in terms of the $W$ symbols. Therefore, different $W$ symbols in $H_{x_{j}}$ have different $x$ generators, aside from $x_{j}$ (for $\left.j=1,2, \cdots, r\right)$.

Further, if $W=W\left(\theta, x_{j}\right) \in H_{x_{j}}$ then $\left\langle W_{1}, \cdots, W_{r}, W\left(\theta, x_{j}\right)\right\rangle=\left\langle x_{1}, x_{2}, \cdots, x_{k}, \theta\right\rangle$ (always appealing to Theorem 2.2 ) so that

$$
W\left(\theta, x_{j}\right)=x_{j}^{k(W)} \theta^{\epsilon(W)} x_{j}^{l(W)}
$$

where $k(W), l(W)$ are integers and $\epsilon(W)= \pm 1$. This shows that $H_{x_{j}}$ consists of $W$ symbols of the type (i), for $j=1,2, \cdots, r$.

From the expression (i) we find that

$$
L_{W}(\theta)=L_{W}\left(x_{j}^{-k(W)}\right)+L_{W}\left(x_{j}^{-l(W)}\right)+1 \geq L_{W}\left(x_{j}\right)+1 \geq 2 .
$$

By using the standard finite Nielsen transformations we may convert $\left\{W_{1}\right.$, $\left.W_{2}, \cdots, w_{r}\right\}$ to $\left\{x_{1}, x_{2}, \cdots, x_{r}\right\}$ in a finite number of steps and then applying the elementary simultaneous transformations applied on the sets $H_{x_{1}}, H_{x_{2}}, \cdots, H_{x_{r}}$, using $\left\{x_{1}, x_{2}, \cdots, x_{r}\right\}$ as "active" elements (here $x_{j}^{ \pm 1}$ would be applied to $H_{x_{j}}$ in the appropriate manner), we may in a finite number of steps convert $\left\{W_{1}, W_{2}\right.$, $\left.\cdots, W_{r}\right\} \cup H_{x_{1}} \cup \cdots \cup H_{x_{r}}$ to the set of $x$ generators $\left\{x_{1}, \cdots, x_{r}\right\} \cup \bar{H}_{x_{1}} \cup \cdots$ $\cup \bar{H}_{x_{r}}$. Here $\bar{H}_{x_{j}}=\left\{\theta \in\left\{x_{\nu}\right\} \mid \theta \neq x_{j}\right.$ and $\theta$ occurs in $\left.W \in H_{x_{j}}\right\}$, for $j=1,2, \cdots, r$.

Let $\Sigma_{1}=\left\{x_{1}, \cdots, x_{r}\right\} \cup\left(\bigcup_{j=1}^{r} \bar{H}_{x_{j}}\right)$ and set $H_{1}=\bigcup_{j=1}^{r} H_{x_{j}}, H_{2}=$ $\left\{W_{\nu} \mid W_{\nu} \notin\left\{W_{1}, \cdots, W_{r}\right\} \cup H_{1}\right.$, and $W_{\nu}$ contains an $x$ generator from $\left.\bigcup_{j=1}^{r} \bar{H}_{x_{j}}\right\}$. Now if $V \in H_{2}$ then $V=V(\theta, \xi)$, where $\theta \in \bigcup_{j=1}^{r} \bar{H}_{x_{j}}$ and $\xi \notin \Sigma_{1}$. Just as before, it follows that $L_{W}(\xi) \geq L_{W}(\theta)+1 \geq 2+1=3$. Again, applying elementary simultaneous Nielsen transformations to the set $\Sigma_{1} \cup H_{2}$ we may convert this set to $\Sigma_{2}$ which consists of only $x$ generators $\left(\Sigma_{2} \supset \Sigma_{1}\right)$.

Set $H_{3}=\left\{W_{\nu} \mid W_{\nu} \notin\left\{W_{1}, \cdots, W_{r}\right\} \cup H_{1} \cup H_{2}\right.$, and $W$ contains an $x$ generator from $\left.\Sigma_{2}\right\}$. Here, if $U \in H_{3}$, then $U=U(\phi, \gamma)$ where $\phi \in \Sigma_{2}$ and $\gamma \notin \Sigma_{2}$. It then follows that $L_{W}(\gamma) \geq L_{W}(\phi)+1 \geq 3+1=4$.

More generally, if $H_{l}=\left\{W_{\nu} \mid W_{\nu} \notin\left\{W_{1}, \cdots, W_{r}\right\} \cup H_{1} \cup \cdots \cup H_{l-1}\right.$ and $W_{\nu}$ contains an $x$ generator of $\left.\Sigma_{l-1}\right\}$ then, for $W \in H_{l}, W=W(\zeta, \lambda)$ where $\zeta \in \Sigma_{l-1}$ and $L_{W}(\lambda) \geq L_{W}(\zeta)+1 \geq l+1$. $\Sigma_{l}$ is defined as the set of $x$ generators when $\Sigma_{l-1} \cup H_{l}$ is reduced by elementary simultaneous transformations $\left(\Sigma_{l} \supset \Sigma_{l-1}\right)$.

Thus the sequence $\left\{w_{1}, \cdots, w_{r}\right\}, H_{1}, H_{2}, \cdots, H_{l}, \cdots$ will break off before the $N$ th stage of construction, say, at the Mth stage where $M<N$. Also the set $\left\{W_{1}, \cdots, w_{k}\right\} \cup H_{1} \cup H_{2} \cup \cdots \cup H_{M}$ may be converted to the set $\Sigma_{M}$ by a finite 
application of elementary simultaneous Nielsen transformations.

We set $X_{1}=\Sigma_{M}$, and suppose that $X_{j}$ has been defined for all $j<k$, for some $k$. We define $X_{k}$ as follows:

(a) First, let $\bar{X}_{k}=\bigcup_{j<k} X_{j}$.

(b) Pick a generator $x^{*} \in\left\{x_{\nu}\right\}-\bar{X}_{k}$ and repeat in an analogous manner the construction of the set $X_{1}$ by use of $x_{1}$, but now use $x^{*}$ in place of $x_{1}$. Call this set $X_{k}$.

It is evident, from the manner of construction, that the groups $\left\langle X_{k}\right\rangle$ are pairwise disjoint, i.e., intersect in the identity. They are generated by disjoint subsets of the free generators $\left\{x_{\nu} \mid \nu \in \mathcal{N}\right\}$.

Let the construction terminate at some ordinal number $\tau$. Then, $F={ }^{*} \Pi_{k \leq r} X_{k}$ (free product). We may certainly find a permutation $\pi: x_{\nu} \rightarrow x_{\pi(\nu)}$ such that $\left\langle\alpha \pi\left(X_{k}\right)\right\rangle=\left\langle X_{k}\right\rangle$ and where $\left.\alpha \pi\right|_{X_{k}}$ is a finite product of elementary simultaneous Nielsen automorphisms (since $\alpha \pi\left(X_{k}\right)$ may be converted to a system of $x$ generators by a finite application of elementary simultaneous Nielsen automorphisms).

The number of elementary simultaneous Whitehead transformations used to convert $\left\{W_{1}, \cdots, W_{r}\right\} \cup H_{1} \cup \cdots \cup H_{M}$ to a set of $x$ generators can be given an upper bound depending only on $N$. This upper bound can be used to estimate the number of these automorphisms used to produce $\alpha$.

To reduce the set $\left\{W_{1}, \cdots, w_{r}\right\}$ to $\left\{x_{1}, \cdots, x_{r}\right\}$ will require no more than $L_{x}\left(W_{1}\right)+\cdots+L_{x}\left(W_{r}\right)-r$ elementary Whitehead transformations (this follows from J. H. C. Whitehead's results [8], [9] or see [3, pp. 166-167]). Since $r$ and $L_{x}(W)$ are both no more than $N$, the reduction process will require no more than $\mathrm{Nr}-r$ Whitehead transformations. In reducing the set $H_{1} \cup H_{2} \cup \cdots \cup H_{M}$ we are applying no more than $M$ simultaneous transformations, where $M<N$. Thus the smallest number $\bar{N}$ of elementary transformations required to convert the $W$ 's to the $x$ 's is no more than $N(N-1)+M+1$ (if we include the permutation with exponent sign), and therefore $\bar{N}<N(N-1)+N+1=N^{2}+1$.

\section{REFERENCES}

1. M. Hall, Jr., The theory of groups, Macmillan, New York, 1959. MR 21 \#1996.

2. F. W. Levi, Über die Untergruppen der freien Gruppen, Math. Z. 32 (1930), 315-318.

3. W. Magnus, A. Karrass and D. Solitar, Combinatorial group theory: Presentations of groups in terms of generators and relations, Pure and Appl. Math., vol. 13, Interscience, New York, 1966. MR 34 \#7617.

4. J. Nielsen, Om Regnig med ikke-kommutative Faktorer og dens Anvendeise $i$ Gruppeteorien, Mat. Tidsskr. B 1921, 77-94.

5. —- Die Isomorphismengruppe d., freien Gruppen, Math. Ann. 91 (1924), 169209.

6. E. S. Rapaport, On free groups and their automorphisms, Acta Math. 99 (1958), 139-163. MR 24 \#A1302. 
7. D. H. Wagner, On free products of groups, Trans. Amer. Math. Soc. 84 (1957), 352-378. MR 18, 790.

8. J. H. C. Whitehead, On certain sets of elements in a free group, Proc. London Math. Soc. 41 (1936), 48-56.

9. - On equivalent sets of elements in a free group, Ann. of Math. 37 (1936), $782-800$.

DE PARTMENT OF MATHEMATICS, YORK COLLEGE, CITY UNIVERSITY OF NEW YORK, JAMAICA, NEW YORK 11432 